\title{
Identification of Novel Long Noncoding RNAs Associated with TGF- $\beta /$ Smad3-Mediated Renal Inflammation and Fibrosis by RNA Sequencing
}

\author{
Qin Zhou, ${ }^{* \dagger}$ Arthur C.K. Chung, ${ }^{* \dagger}$ Xiao R. Huang, ${ }^{\star}$ Yuan Dong, ${ }^{*}$ Xueqing Yu, ${ }^{\dagger}$ and Hui Y. Lan ${ }^{* \dagger}$
}

\begin{abstract}
From the Department of Medicine and Therapeutics, * the Li Ka Shing Institute of Health Sciences, The Chinese University of Hong Kong, Hong Kong; the Department of Nephrology, ${ }^{\dagger}$ The First Affiliated Hospital, Sun Yat-sen University, Guangzhou; and the Chinese University of Hong Kong Shenzhen Research Institute, ${ }^{\ddagger}$ Shenzhen, China
\end{abstract}

\author{
Accepted for publication \\ October 11, 2013. \\ Address correspondence to \\ Hui Y. Lan, M.D., Ph.D., \\ Department of Medicine and \\ Therapeutics, Li Ka Shing \\ Institute of Health Sciences, The \\ Chinese University of Hong \\ Kong, Shatin, The New Terri- \\ tories, Hong Kong, China; or to \\ Xueqing Yu, M.D., Ph.D., \\ Department of Nephrology, The \\ First Affiliated Hospital, Sun \\ Yat-sen University, 58th Zhong- \\ shan Rd II, Guangzhou 510080, \\ China. E-mail: hylan@cuhk. \\ edu.hk or yuxq@mail.sysu.edu. \\ cn.
}

\begin{abstract}
We have previously shown that transforming growth factor- $\beta / S$ mad3-dependent miRNAs play a critical role in renal inflammation and fibrosis. However, off-target effects of miRNAs limit their therapeutic application. Recently, emerging roles of long noncoding RNAs (IncRNAs) in diseases have been recognized. In this study, we used high-throughput RNA sequencing to identify the Smad3-dependent IncRNAs related to renal inflammation and fibrosis in Smad3 knockout mouse models of unilateral ureteral obstructive nephropathy and immunologically induced anti-glomerular basement membrane glomerulonephritis. Compared with wild-type mice, 151 IncRNAs in the unilateral ureteral obstructive nephropathy kidney and 413 IncRNAs in kidneys with anti-glomerular basement membrane glomerulonephritis were significantly altered in Smad3 knockout mice. Among them, 21 common IncRNAs were up-regulated in wildtype, but down-regulated in Smad3 knockout, kidneys in both disease models in which progressive renal inflammation and fibrosis were abolished when the Smad3 gene was deleted or suppressed. Realtime PCR confirmed these findings and revealed the functional link between Smad3-dependent IncRNAs np_5318/np_17856 and progressive kidney injury. Results demonstrate that the identification and characterization of functional IncRNAs associated with kidney disease may represent a promising research direction into renal disorder and may lead to the development of new IncRNA therapies for kidney diseases. (Am J Pathol 2014, 184: 409-417; http://dx.doi.org/10.1016/j.ajpath.2013.10.007)
\end{abstract}

Increasing evidence shows that chronic kidney disease (CKD) is a major problem and health care burden worldwide. More than $10 \%$ of the entire population experiences different stages of CKD. Renal inflammation and fibrosis are the common manifestations of CKD. ${ }^{1}$ Transforming growth factor- $\beta 1$ (TGF- $\beta 1$ ), a well-studied profibrogenic cytokine, exerts its biological effects by activating its downstream mediators, Smad2 and Smad3, which is counterregulated by an inhibitory Smad7. ${ }^{2,3}$ Although Smad3 is a key transcription factor in response to many fibrogenic mediators, targeting Smad3 may cause autoimmune disease by impairing immunity. ${ }^{2,4-6}$ Thus, alternative approaches to inhibit TGF- $\beta$ actions should be developed in an attempt to suppress renal inflammation and fibrosis without impairing the immune system.

Emerging evidence shows that noncoding RNAs (ncRNAs) may play a critical role in the development of kidney diseases.
The identification of disease-related miRNAs in nephropathy was thought to be a specific therapy for treating the kidney disease. $^{3,7-16}$ However, the off-target effects hinder the development of specific miRNA therapy, because one miRNA is able to regulate many target genes. 7,10

Since the discovery of Xist, a long noncoding RNA (lncRNA) required for mammalian $\mathrm{X}$ chromosome inactivation, ${ }^{17}$ many

\footnotetext{
Supported by the Major State Basic Research Development Program of China, 973 program, grant 2012CB517700 (H.Y.L.) and the National Natural Science Foundation of China, Major Program grant 81130012 (X.Y.), General Program grant 81170681 (A.C.K.C.), Young Scientists Fund grant 81100542 (Q.Z.), the Research Grant Council of Hong Kong grants RGC GRF 468711, CUHK5/CRF/09, and CUHK3/CRF/12R (H.Y.L.) and GRF 464010 and 463612 (A.C.K.C.), and the Focused Investment Scheme A and B from the Chinese University of Hong Kong.

Q.Z. and A.C.K.C. contributed equally to this work.
} 
lncRNAs have been reported in mammals and other vertebrates. ${ }^{18}$ At present, many lncRNAs, defined as RNAs $>200$ nucleotides (nt) that do not encode any protein, are found to be able to control gene expression. ${ }^{18}$

The development of high-throughput RNA sequencing (RNA-Seq) accelerates the discovery of novel ncRNAs because RNA-Seq can achieve single-base resolution and capture all of the transcripts in the samples. ${ }^{19,20}$ In this study, we used this technique to search for Smad3dependent lncRNAs during renal injury in mouse models of immunologically induced anti-glomerular basement membranous glomerulonephritis (anti-GBM GN) and nonimmune disease of unilateral ureteral obstructive nephropathy (UUO). The results of real-time PCR validation, sequence analyses, and chromatin immunoprecipitation (ChIP) assays revealed a functional link between Smad3dependent lncRNAs and progressive kidney injury. Thus, outcomes from this study suggested a novel research direction into renal disorder and may promote new therapeutic strategies for kidney diseases by targeting lncRNAs.

\section{Materials and Methods}

\section{Mouse Model of UUO and Anti-GBM GN}

The animal models of UUO and anti-GBM GN were induced in both sexes of C57BL/6J wild-type (WT) and Smad3 knockout (KO) mice at 8 to 10 weeks of age, as previously described. $^{21,22}$ Briefly, for the UUO model, the left ureter from Smad 3 WT or KO mice $(n=8$ per group, both sexes, 8 weeks of age, 22 to $25 \mathrm{~g}$ body weight) was ligated and the
UUO kidney was harvested at day 5 after surgery for further analysis. In addition, a group of eight $\operatorname{Smad} 3$ WT or KO mice, sham operated on, were used as a control. For the anti-GBM GN model, groups of eight Smad3 WT or KO mice were preimmunized with sheep IgG in Freund's complete adjuvant (Sigma Chemical Co, St. Louis, MO), followed 5 days later by i.v. administration of sheep anti-mouse GBM immunoglobulin at a dose of $60 \mu \mathrm{g} / \mathrm{g}$ of body weight (termed day 0 ). Diseased mice were euthanized at day 10 for further analysis. Groups of age- and weight-matched normal Smad3 WT or KO mice were used as normal controls $(n=8)$. The experimental procedures for both models were performed following the approved protocol by the Animal Experimentation Ethics Committee at The Chinese University of Hong Kong (Hong Kong).

\section{Ultrasound-Mediated Gene Transfer of Inducible Smad7 Overexpression Plasmids into the Ligated Kidneys}

The mixture of Smad7 and tetracycline-on (tet-on) plasmids was transferred into the ligated kidneys of UUO, as described previously. ${ }^{23}$ The experimental procedures were approved by the Chinese University of Hong Kong's Animal Experimental Ethics Committee.

\section{Smad7 Knockout Mice}

Mutant mice deficient in exon 1 of the $\operatorname{Smad} 7$ gene from a CD1 background (provided by Prof. Rainer L. Heuchel,

Table 1 Real-Time PCR Primers for 21 lncRNAs and GAPDH

\begin{tabular}{|c|c|c|}
\hline Gene & Forward primer & Reverse primer \\
\hline np_18588 & $5^{\prime}$-CGGAGGATTGATAGCCAAAA-3' & $5^{\prime}$-CCTGGTTGTCGTGTTTCCTT-3' \\
\hline np_26992 & $5^{\prime}$-GACCAATGTTCGTAGCAGCA-3' & 5'-тССтTCTCTTTGTGCGTGTG-3' \\
\hline na_2414 & 5'-CTGCCTCTGTGCTGCATTTA-3' & $5^{\prime}$-TCTTTGTCACCCAAGCAGTG-3' \\
\hline np_29908 & 5'-TGGATTTGGCCTCAGTTTTC-3' & 5'-CAGCTTCGAGTTTGTCACCA-3' \\
\hline np_16555 & 5'-CAGCCTAGCTCACCTTCTGG-3' & 5'-TGTTCATCTGTGGCTCTTGG-3' \\
\hline np_5340 & $5^{\prime}$-TTCCTGGGCTTCACAAGTTT-3' & 5'-GCGTGGCTTCAGGTATTTGT-3' \\
\hline np_17234 & 5'-TGTTTTAACCСАTTGTATCATTTCA-3' & $5^{\prime}$-CAACCCCCTTTGGAAGACTC-3' \\
\hline np_18603 & 5'-CCTTTACAAGCAAAAGCCAAA- 3' & $5^{\prime}$-CCCTTGAGACACTGCCTGAC-3' \\
\hline na_9884 & $5^{\prime}-\mathrm{ACCACAGCAGATACCCAAGC-3^{ \prime }}$ & $5^{\prime}$-CAGCAAGCTCCTTTTTCCAC-3' \\
\hline np_5392 & $5^{\prime}$-GGGTGTGAACGGAGACTGTT-3' & 5'-GGCGAGGAATGTGTTTGTTT-3' \\
\hline np_18004 & $5^{\prime}$-CTCCATAAAGAGCCCCATGA-3' & $5^{\prime}-\mathrm{TTGGGCCTTCCAACAATAAC-3^{ \prime }}$ \\
\hline np_28730 & 5'-TCTGGGTCCAАСTCCАTTTT-3' & 5'-CCTGTTTTAATTGGGGGAAA-3' \\
\hline np_4334 & $5^{\prime}$-AGAGGGCAAGCAACATTGTC-3' & $5^{\prime}$-GCTGTGGGGAGAGCAACTAA-3' \\
\hline np_18636 & 5'-CAACCTAAGGCTGCTCTCTCC-3' & $5^{\prime}$-CCATCAAGAAACCTGGCAAT-3' \\
\hline np_17856 & $5^{\prime}$-GAAGCCTGCAGGAAGATGAC- $3^{\prime}$ & $5^{\prime}-\mathrm{ACCAGTCACAAAAGCCAACC-3^{ \prime }}$ \\
\hline np_18537 & 5'-TTCCAGTAGTGCTGCACAGG-3' & 5'-TCCAGGTTTCCAACTTTTGC-3' \\
\hline np_5318 & $5^{\prime}-\mathrm{AACTCGCCACAGAAATCCAC-3^{ \prime }}$ & $5^{\prime}-\mathrm{ACAACCCCAAACAAGCTGTC-3^{ \prime }}$ \\
\hline np_19902 & 5'-TGTTGCTAGAATGGCAGGTG-3' & 5'-TCCTGCCTGGGAATACTGTC-3' \\
\hline np_28233 & 5'-GATGGGGAAGAAACCCATTT-3' & 5'-CAGGGCCAGTTATGTCCAGT-3' \\
\hline np_2108 & 5'-AGATCTCCССTCTTGGGAAA-3' & $5^{\prime}$-СТСССТСТССАСАСТGСТTC-3' \\
\hline np_28496 & $5^{\prime}$-AGCAGGCTAAGTCAGGGTGA-3' & 5'-GGGTTGAGCTGCCATGTATT-3' \\
\hline GAPDH & 5'-TGCTGAGTATGTCGTGGAGTCTA-3' & $5^{\prime}$-AGTGGGAGTTGCTGTTGAAATC-3' \\
\hline
\end{tabular}


Karolinska Institutet, Stockholm, Sweden) have been described recently. ${ }^{24}$ All mice were from a CD1 background. A mouse model of UUO was induced in groups of six to eight $\operatorname{Smad} 7 \mathrm{KO}$ or WT mice (male, 8 weeks of age, 22 to $25 \mathrm{~g}$ body weight), as previously described. ${ }^{21,25}$ The experimental procedures were approved by the Chinese University of Hong Kong's Animal Experimental Ethics Committee.

\section{RNA Preparation for Sequencing}

Kidney tissues of Smad3 KO/WT mice $(n=2$ in each group) were collected from a normal, UUO model at day 5 , and an anti-GBM GN model at day 10 for RNA sequencing. Total RNA from the kidney tissue was extracted by TRIzol reagent (Life Technologies, Carlsbad, CA), as per the manufacturer's instructions. Additional DNase I digestion and clear-up steps were performed per the protocol from the Beijing Genomics Institute (Shenzhen, China). RNA quality and purity were determined by the ND-1000 Nanodrop (NanoDrop, Wilmington, DE). For each sample, RNAs were fractionated with a denaturing polyacrylamide gel. To maximize the ncRNA database for analysis, RNAs $>150 \mathrm{nt}$ were used for cDNA synthesis. After adenylating the $3^{\prime}$ end of RNAs and ligating the adapter, the RNA samples were preceded to library construction following the protocol at Beijing Genomics Institute (Supplemental Figure S1). The sequencing was performed by the Genome Analyzer (serial No. Hiseq2000; Illumina, San Diego, CA).

\section{Analysis of Sequencing Data and Assembling RNA Transcripts}

Low-quality reads, $>50 \%$ of the sequence reads (including adapters) with a quality score of $\leq 10$ and $N>5 \%$, were discarded. Ribosome RNA sequences were filtered from the raw fragments. Remaining clean reads were mapped to a genome (University of California, Santa Cruz, CA, genome
A

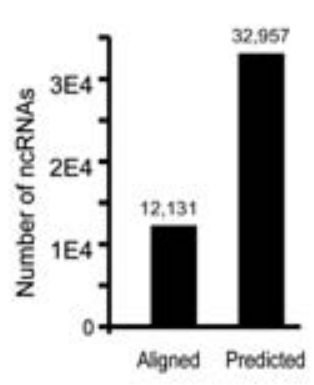

B

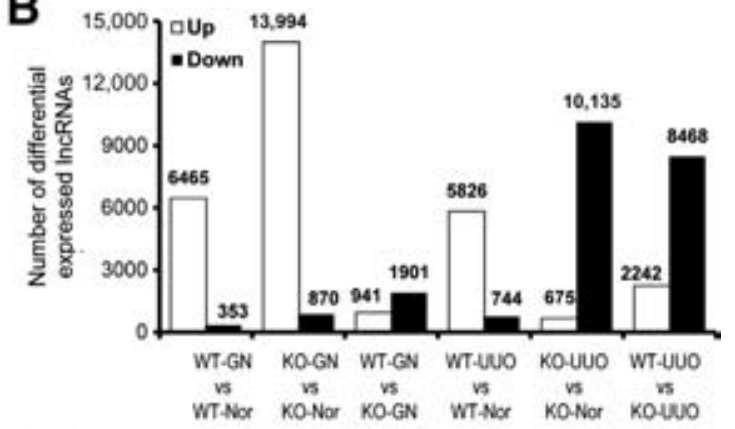

C uUo

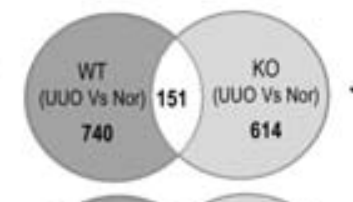

WTar RONAT KO-GN
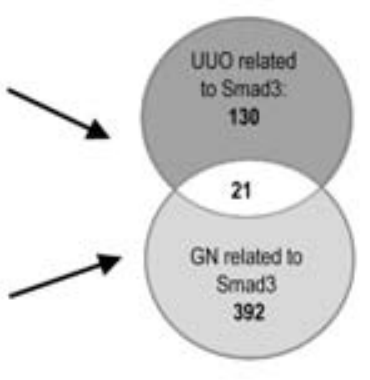

D
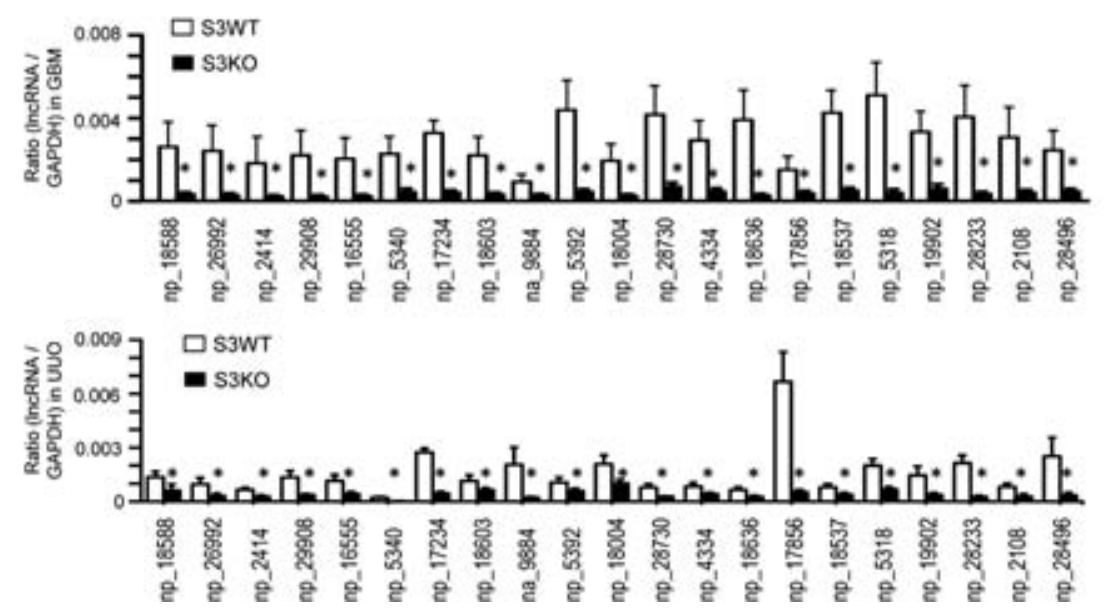

Figure 1 Results of RNA-Seq in Smad3 WT/KO kidneys in UUO and anti-GBM GN models. A: The number of transcripts aligned to the known ncRNA database and transcripts predicted to be ncRNAs. B: Comparisons of differentially expressed IncRNAs (length $>200 \mathrm{nt}$ ) between normal and diseased kidney and between Smad3 KO and WT mice in two kidney disease models. C: Venn diagrams demonstrate overlapped IncRNAs (common IncRNAs) versus nonoverlapped IncRNAs related to Smad3 that were differentially expressed in both UU0 and anti-GBM GN models. $P<0.01$ (B and C). D: Realtime PCR validation of 21 common IncRNAs in antiGBM and UUO models of Smad3 WT (S3WT)/Smad3 $\mathrm{KO}$ (S3K0) mice ( $n=8$ in each group). The relative levels of target IncRNAs were calculated as the ratio to GAPDH. ${ }^{*} P<0.05$ versus S3WT mice. Nor, normal. 
browser, mouse genome July 2007, mm9) by using Tophat [1.3.0.Linux_x86_64 (bowtie-0.12.7)] and then assembled with Cufflinks (1.3.0.Linux_x86_64; Berkeley, CA). To discriminate coding from noncoding transcripts, a support vector machine-based classifier (coding potential calculator) was used to predict novel ncRNAs. ${ }^{26}$ The prediction of novel IncRNA was assembled based on the SwissProt database (Hinxton, Cambridge, UK). All of the ncRNAs were annotated by aligning the genomic sequence against RFAM, a collection of multiple sequence alignments and covariance models representing ncRNA families (http:// rfam.sanger.ac.uk, last accessed August 31, 2013), as previously described. ${ }^{27}$ The RPKM value was used to obtain a comparable measure of gene expression, and Pearson correlation was used to analyze RPKM value correlation. ncRNAs > $200 \mathrm{nt}$ were selected as lncRNAs for further analysis because approximate $200 \mathrm{nt}$ is the common cutoff to define lncRNA in the literature. ${ }^{18}$ The sequencing data obtained were deposited in the National Center for Biotechnology Information database (http://www.ncbi.nlm.nih.gov/ bioproject/223210; accession number PRJNA223210).

\section{Chromosomal Location and Gene Ontology of IncRNAs}

We used an evolutionary conserved region (ECR) browser ${ }^{28}$ to detect any conserved Smad binding motif prediction in mouse regulatory regions. Chromosomal location and gene ontology of the 21 common lncRNAs and their linked genes were searched on the EMBL-EBI database (http://www.ebi.ac.uk, last accessed August 31, 2013) and the ECR Browser (http:// ecrbrowser.dcode.org, last accessed August 31, 2013). ${ }^{28}$

\section{ChIP Analysis}

ChIP was performed with a transcription factor ChIP kit, as previously described. ${ }^{15,29}$ In brief, cells were cross-linked with $1 \%$ formaldehyde for 10 minutes at $37^{\circ} \mathrm{C}$, quenched with glycine, and then sonicated using a Bioruptor (Diagenode, Liège, Belgium) to generate 300- to 600-bp DNA fragments. Immunoprecipitation was performed with the antibody against Smad3 (Upstate/Millipore, Billerica, MA), and a normal IgG was used as a control. Precipitated DNAs were detected by PCR using specific primers: Smad binding site (SBS) for np_17856, 5'-GAAGAGATCTTATACATTTCCCCCTA- $3^{\prime}$ and $5^{\prime}$-TTCCTCTTCCCAATGTGGTC- $3^{\prime}$; and SBS for np_5318, 5'-CTCTCTCAAACAGCCTGTGG-3' and $5^{\prime}$-GAAATTTGGAGGTGCAATCAA-3'.

\section{RT-PCR and Real-Time PCR Analysis}

lncRNA expression was quantified by real-time PCR with SYBR Green (Life Technologies, Carlsbad, CA) as the fluorescent reporter. Total RNA $(1 \mu \mathrm{g})$ was used to synthesize the first strand of cDNA using M-MLV Reverse Transcriptase (Life Technologies). The real-time PCR was performed as previously described. ${ }^{23}$ Primers for 21 common lncRNAs and glyceraldehyde-3-phosphate dehydrogenase (GAPDH) are listed in Table 1. The relative levels of target

Table 2 Comparison of Expression Levels of 21 Common IncRNAs in S3WT/S3KO Kidneys with UUO and Anti-GBM GN

\begin{tabular}{|c|c|c|c|c|c|}
\hline \multirow[b]{2}{*}{ IncRNA } & \multirow[b]{2}{*}{ Length (nt) } & \multicolumn{4}{|l|}{$\log _{2}$ fold change } \\
\hline & & S3WT (UU0 vs Nor) & S3K0 (UU0 vs Nor) & S3WT (GBM vs Nor) & S3KO (GBM vs Nor) \\
\hline np_18588 & 574 & $2.146^{*}$ & $-3.660 * *$ & $2.834 * * *$ & $-4.600 * *$ \\
\hline na_2414 & 528 & $2.196 * *$ & $-3.245^{\star \star \star}$ & $2.320 * *$ & $-2.085^{\star * *}$ \\
\hline np_29908 & 1413 & $2.196 * *$ & $-4.075^{* *}$ & $2.213^{* *}$ & $-5.015^{\star * *}$ \\
\hline np_16555 & 818 & $2.229 * *$ & $-4.245^{* \star *}$ & $3.132 * * *$ & $-5.185^{\star \star *}$ \\
\hline np_18603 & 606 & $2.381^{\star}$ & $-5.535^{* * *}$ & $2.497 * *$ & $-2.474^{* *}$ \\
\hline na_9884 & 436 & $2.474^{*}$ & $-2.175^{\star * \star}$ & $3.156^{* * *}$ & $-3.337^{* * *}$ \\
\hline np_5392 & 2917 & $2.544 * * *$ & $-2.075^{\star *}$ & $3.320 * * *$ & $-5.600^{* * *}$ \\
\hline np_18004 & 621 & $2.644 * * *$ & $-3.883^{* *}$ & $2.294 * * *$ & $-4.822^{* *}$ \\
\hline np_28730 & 436 & $2.703 * * *$ & $-4.776^{* *}$ & $2.227^{* * *}$ & $-4.715^{\star *}$ \\
\hline np_5318 & 2180 & $3.392 * * *$ & $-2.798 * \star *$ & $3.642 * * *$ & $-6.059 * * *$ \\
\hline np_19902 & 747 & $3.917 * * *$ & $-4.776^{* *}$ & $3.589 * * *$ & $-4.715^{\star *}$ \\
\hline np_28233 & 1508 & $4.428^{* * *}$ & $-4.660 * *$ & 3.419 & $-4.600^{* *}$ \\
\hline np_2108 & 737 & $5.381 * * *$ & $-3.535^{* * *}$ & $4.642 * *$ & $-2.474^{* *}$ \\
\hline np_28496 & 658 & $6.146^{* * *}$ & $-2.982^{* *}$ & $5.004^{* * *}$ & $-4.922^{* *}$ \\
\hline
\end{tabular}

${ }^{*} P<0.01,{ }^{* *} P<0.001$, and ${ }^{* * *} P<0.0001$ for the comparison between groups.

Nor, normal; S3K0, Smad3 K0; S3WT, Smad3 WT. 
lncRNAs were calculated using the $\Delta \Delta \mathrm{C}_{\mathrm{T}}$ method, and the data were normalized with GAPDH.

\section{Statistical Analysis}

For IncRNA expression by RNA-Seq, significance levels were indicated as $P<0.01$ and greater than twofold change. Data obtained from real-time PCR are expressed as the means \pm SEM and analyzed using a $t$-test in Microsoft Excel (Redmond, WA).

\section{Results}

In this study, high-throughput RNA-Seq was used to search for Smad3-dependent lncRNAs during renal injury in mouse models of anti-GBM GN and UUO. Kidneys of Smad3 KO/ WT mice with UUO and anti-GBM GN were collected for sequencing, as described in Supplemental Figure S1. Total RNA of all 12 samples of Smad3 KO/WT kidneys was sequenced by Illumina, each generating $>50$ million reads. Low-quality reads and rRNA sequences were filtered from the raw fragments. Total clean reads were then mapped to genome by using Tophat, an alignment program that maps RNA-Seq reads across the splice junction without relying on gene annotation. ${ }^{30}$ As shown in Supplemental Table S1, the proportion of clean reads that mapped to the genome ranged from $71.55 \%$ to $85.82 \%$ in 12 samples. The mapped reads were assembled into transcripts with Cufflinks, putative transcripts containing both coding and noncoding transcripts. ${ }^{31}$

The identification and characterization of lncRNAs are an experimental and computational challenge. In the present study, all assembled transcripts were aligned to a ncRNA database using blastn $\left(1 \times 10^{-10}\right)$. The filter was set as identity $>0.9$ and coverage $>0.8$. Reads mapped to multinoncoding RNA databases using the previously described criteria were identified as noncoding RNAs. Approximately $20.03 \%$ to $35.71 \%$ clean reads were mapped to the ncRNA database (obtained from frnadb, lncrnadb, NONCODE, RNAdb, and ensembl) (Supplemental Table S1). All ncRNAs were then annotated by aligning a genomic sequence against RFAM, a collection of multiple sequence alignments and covariance models representing ncRNA families. The resulting hits were then used as supporting evidence for ncRNAs. At last, 12,131 transcripts were aligned to the known ncRNA database, and 32,957 transcripts were predicted to be ncRNAs with a length of $>150$ nt (Figure 1A).

We next identified lncRNAs that differentially expressed, in both models of anti-GBM GN and UUO kidneys, the transcript abundances measured by reads per kb per million $\left(\mathrm{RPKM}\right.$; RPKM $\left.=10^{9} \times \mathrm{C} / \mathrm{NL}\right)$ where $\mathrm{C}$ is the reads number of the transcript, $\mathrm{L}$ is the length of the transcript, and $\mathrm{N}$ is the total reads number of the sample (Supplemental Table S2). ${ }^{32}$ Pearson correlation analysis showed that the RPKM values between these groups correlated moderately

Table 3 Chromosomal Location and Gene Ontology of 21 Common IncRNAs and Their Linked Genes

\begin{tabular}{|c|c|c|c|c|}
\hline IncRNA & Status & Chromosomal location & Linked gene & Gene Ontology* \\
\hline np_18588 & Intronic & Chr1: 55, 627, 209-55, 627, 782 & Plcl1 & Signal transducer activity and lipid metabolic process \\
\hline np_26992 & Intronic & Chr10: 40, 123, 552-40, 124, 779 & Slc16a10 & Transmembrane transport \\
\hline np_29908 & Intronic & Chr1: 55, 657, 642-55, 659, 054 & Plcl1 & Signal transducer activity and lipid metabolic process \\
\hline np_16555 & Intronic & Chr2: $135,078,612-135,079,429$ & Plcb1 & $\begin{array}{l}\text { Intracellular signal transduction and interleukin-mediated } \\
\text { signaling pathway }\end{array}$ \\
\hline np_18603 & Intronic & Chr1: $61,800,641-61,801,246$ & Pard3b & Cell cycle, cell division, and tight junction \\
\hline na_9884 & Sense & Chr5: 52, 973, 057-52, 992, 294 & 5033403H07Rik & None \\
\hline np_5392 & Intronic & Chr3: $30,449,176-30,452,092$ & Mecom & Apoptosis, differentiation, and transcription \\
\hline np_18004 & Intronic & Chr16: 51, 132, 993-51, 133, 613 & $T b c 1 d 5$ & Positive regulation of RabGTPase activity \\
\hline np_28730 & Intronic & Chr16: $77,503,372-77,503,807$ & $2810055 G 20 R i k$ & None \\
\hline np_5318 & Intronic & Chr1: $69,073,523-69,075,702$ & Erbb4 & Apoptosis, lactation, and transcription \\
\hline np_19902 & Intergenic & Chr7: $37,982,813-37,983,559$ & Tshz3 & Regulation of transcription and DNA dependent \\
\hline np_28233 & Intronic & Chr14: 22, 305, 506-22, 307, 013 & $1700112 E 06 R i k$ & None \\
\hline np_2108 & Intergenic & Chr1: $133,222,856-133,223,592$ & $P P P 1 R 15 B$ & $\begin{array}{l}\text { Translational initiation, signal transduction, and } \\
\text { hydrogen peroxide }\end{array}$ \\
\hline np_28496 & Sense & Chr15: 96, 351, 836-96, 352, 493 & ARID2 & $\begin{array}{l}\text { Chromatin modification, nucleosome disassembly, } \\
\text { and regulation of transcription }\end{array}$ \\
\hline
\end{tabular}

*Gene ontology was searched on the EMBL-EBI database (http://www.ebi.ac.uk, last accessed August 31, 2013).

$\mathrm{Chr}$, chromosome. 
(Supplemental Figure S2), which may be due to low expression levels of IncRNAs within the diseased kidney because higher expression levels of mRNAs (0.8 to 1.0$)$ in these diseased kidney tissues were detected by the Pearson correction analysis (data not shown). Further studies revealed that, compared with normal mice, many lncRNAs were differentially expressed in the diseased kidney of Smad3 WT mice, but this was less abundant in Smad3 KO mice (Figure 1B). Interestingly, deletion of Smad3 largely altered the expression levels of lncRNAs in both disease models (Figure 1B). Of these, 151 lncRNAs (plus or minus twofold to eightfold) in UUO and 413 lncRNAs (plus or minus twofold to ninefold) in anti-GBM GN were differentially expressed in WT and Smad3 KO kidneys (Figure 1C). Among them, 21 lncRNAs were altered in both UUO and anti-GBM GN models, being Smad3-dependent common lncRNAs (Figure 1C). Expression levels of these common IncRNAs were up-regulated in WT (plus twofold to approximately sixfold), but suppressed in $\operatorname{Smad} 3 \mathrm{KO}$, mice with UUO ( - twofold to approximately fivefold) and antiGBM GN (-twofold to approximately sixfold) $(P<0.01)$ (Table 2). To confirm the expression levels of these 21 common lncRNAs in vivo, real-time PCR assays were used. The relative expression levels of 21 lncRNAs were significantly up-regulated in WT, but inhibited in Smad3 KO, kidneys in both anti-GBM GN and UUO models (Figure 1D).

According to the relationship between lncRNAs and their associated protein-coding genes, IncRNAs are classified as sense, antisense, intronic, divergent, and intergenic. ${ }^{33,34}$ In the present study, we focused on the 21 common IncRNAs because they shared all features of both anti-GBM GN and UUO models. The genomic status of 21 common Smad3dependent lncRNAs included two sense, two intergenic, and 17 intronic lncRNAs (Table 3). Some of them were clustered closely within the same chromosome, such as np_18588 and np_29908, and np_18636 and np_5318.

Because 17 lncRNAs were intronic, we then determined whether the linked protein-coding genes of these lncRNAs were involved in inflammation and fibrosis. We blasted 21 common Smad3-dependent IncRNAs in the University of California, Santa Cruz, genome browser (December 2011), GRCm38/mm10. The gene ontology terms for their host genes included cell differentiation, interleukin-mediated signaling transduction, and tight junction, which are likely relevant to inflammation and fibrosis (Table 3), and suggest that these IncRNAs may co-express their linked genes during renal injury.

Although it is known that distant transcriptional activators or repressors (trans-acting) play a role in regulating gene expression, IncRNAs are typically co-expressed with their neighboring genes via cis-acting regulation. ${ }^{18,35-37}$ These reports supported the notion that these 21 common lncRNAs may co-express with the linked genes. We have previously reported that there are several Smad3-dependent miRNAassociated renal fibroses. ${ }^{22,23}$ We then examined whether these linked genes were regulated by Smad 3 by determining any conserved Smad or Smad3 binding sites in the promoter region of lncRNAs and linked genes. We used an ECR browser $^{28}$ to detect any conserved Smad binding motif prediction in mouse regulatory regions. Of $21 \operatorname{lncRNAs}, 8$ had at least one Smad3 binding site and 13 had at least one SBS within the 2-kb upstream regions (Table 4). Despite the poorly conserved promoter regions of IncRNAs among species, ${ }^{18,35-37}$ np_17856 and np_5318 contained conserved Smad3 binding sites in both human and mouse genomes (Figure 2, A-C). To confirm this finding, we used a ChIP assay to determine the interaction of $\mathrm{Smad} 3$ with the promoter regions of np_17856 and np_5318 in mouse embryonic fibroblast (MEFs). As shown in Figure 2D, the antibody against Smad3 could successfully immunoprecipitate the DNA fragments from MEFs containing the potential SBSs in the promoter regions of np_17856 and np_5318, supporting that Smad 3 could physically interact with their promoter regions.

To examine the potential relationship between expression of these Smad3-dependent IncRNAs and progressive renal injury, we validated their expression levels in the UUO kidney from mice with alternation of TGF- $\beta /$ Smad signaling. Consistently, expression of np_5318 and np_17856 in the UUO kidney was both up-regulated in WT mice with severe renal inflammation and fibrosis and abolished in Smad3 KO mice and in mice with overexpression of Smad7 (Figure 2E), in which Smad3 signaling is blocked and renal inflammation

Table 4 Smad Binding Sites in the 2 kb Upstream of 21 Common IncRNAs

\begin{tabular}{|c|c|c|c|}
\hline IncRNA & ECR region (upstream $2 \mathrm{~kb}$ )* & $\begin{array}{l}\text { No. of } \\
\text { Smad3 } \\
\text { binding } \\
\text { sites }\end{array}$ & $\begin{array}{l}\text { No. of } \\
\text { Smad } \\
\text { binding } \\
\text { sites }\end{array}$ \\
\hline np_18588 & Chr1: 55626524-55626634 & 6 & 5 \\
\hline np_26992 & Chr10: 40124874-40124971 & 1 & 1 \\
\hline na_2414 & None & 0 & 0 \\
\hline np_29908 & None & 0 & 0 \\
\hline np_16555 & Chr2: $135077190-135077499$ & 1 & 1 \\
\hline np_5340 & ChrX: 162042604-162042923 & 0 & 3 \\
\hline np_17234 & None & 0 & 0 \\
\hline np_18603 & None & 0 & 0 \\
\hline na_9884 & Chr5: 52994103-52994294 & 0 & 3 \\
\hline np_5392 & Chr3: 30452304-30454092 & 0 & 3 \\
\hline np_18004 & Chr16: 51134121-51134534 & 1 & 0 \\
\hline np_28730 & None & 0 & 0 \\
\hline np_4334 & Chr4: 76297322-76297735 & 2 & 0 \\
\hline np_18636 & Chr1: 68839334-68840018 & 1 & 3 \\
\hline np_17856 & Chr16: 77376543-77376645 & 5 & 5 \\
\hline np_18537 & Chr1: $187857672-187857899$ & 0 & 1 \\
\hline np_5318 & Chr1: 69077396-69077702 & 1 & 2 \\
\hline np_19902 & None & 0 & 0 \\
\hline np_28233 & Chr14: $22304667-22305288$ & 0 & 4 \\
\hline np_2108 & Chr1: $133222674-133222856$ & 0 & 3 \\
\hline Np_28496 & Chr15: 96350567-96350689 & 0 & 2 \\
\hline
\end{tabular}

*The ECR region was searched on the ECR Browser (http://ecrbrowser. dcode.org, last accessed August 31, 2013).

Chr, chromosome. 
A

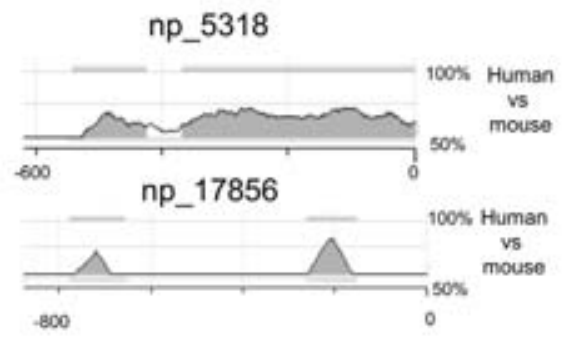

B

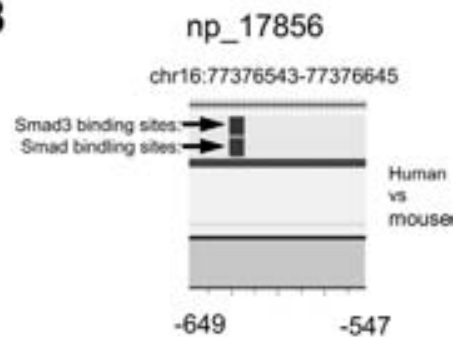

C Smad binding sites:

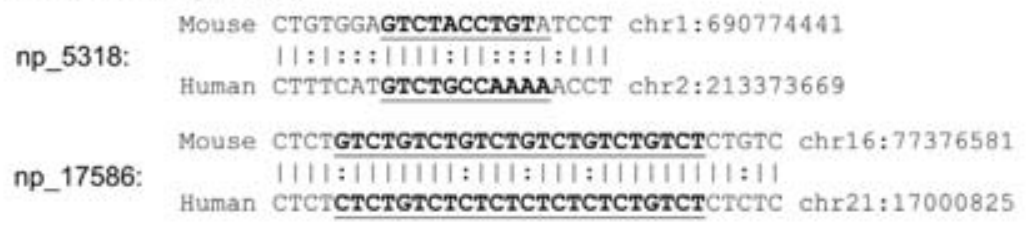

D

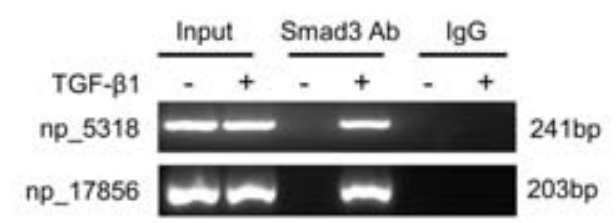

E

Smad3 WT/KO
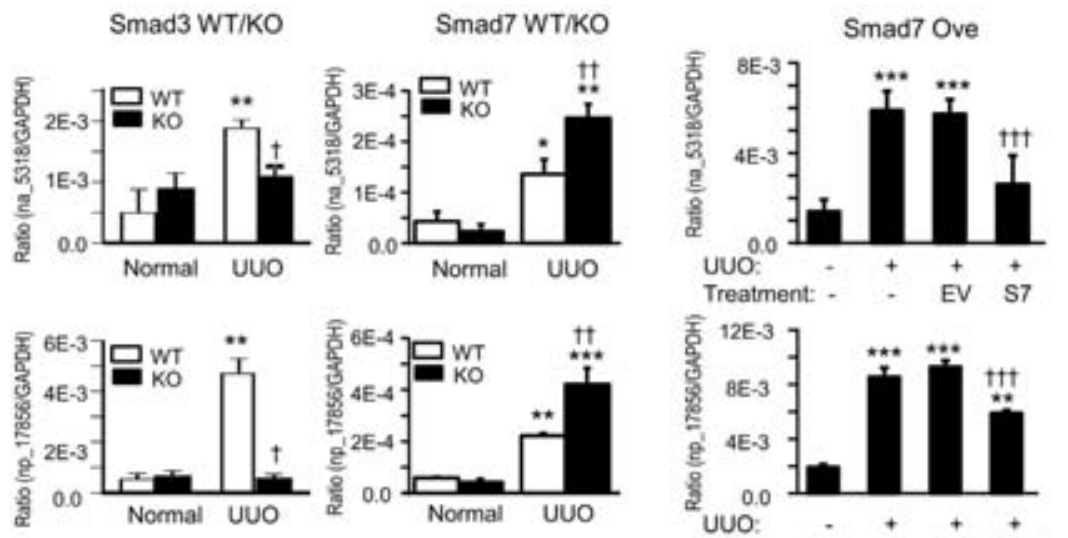

Figure 2 Smad binding sites in the ECR region of two IncRNAs, and expression of two IncRNAs in three different mouse models with alteration of TGF- $\beta /$ Smad signaling. A: Evolutionarily conserved region of np_5318 and np_17856 in human and mouse genomes. B: Conserved Smad3 and Smad binding sites in the ECR region of np_17856. C: DNA sequence alignments of SBS in the promoter regions of np_5318 and np_17856. Bold and underlined sequences indicate the location of SBSs. D: ChIP assays for Smad3 were performed with chromatin from MEF cells treated with TGF- $\beta 1$. Precipitated DNA was amplified with oligonucleotides spanning SBS regions. Total inputs are indicated. E: Real-time PCR results confirm that the expressions of na_5318 and np_17856 are upregulated in ligated kidneys in WT mice, and their expressions are positively correlated with renal injury and activation of TGF- $\beta$ signaling. Each bar represents the means \pm SEM for eight mice. ${ }^{*} P<0.05,{ }^{*} * P<0.01$, and ${ }^{*} * * P<0.001$ versus WT normal mice; ${ }^{\dagger} P<0.05,{ }^{\dagger \dagger} P<0.01$, and ${ }^{\dagger \dagger} P<$ 0.001 versus WT UUO mice. EV, empty vector; S7, Smad7 overexpression (Ove) plasmids; S3WT, Smad3 WT; S3KO, Smad3 KO. The ECR region was searched in the ECR Browser (http://ecrbrowser. dcode.org, last accessed August 31, 2013). ${ }^{28}$ and fibrosis are inhibited. ${ }^{16,23}$ In contrast, expression levels of np_5318 and np_17856 were further enhanced in the UUO kidney of Smad7 KO mice, in which activation of Smad3 signaling and renal inflammation and fibrosis are enhanced, as previously reported. ${ }^{38}$ These results implicate that these Smad3-dependent lncRNAs may have a functional role in the development of renal inflammation and fibrosis, although regulatory mechanisms remain largely unexplored.

\section{Discussion}

TGF- $\beta /$ Smad signaling plays a critical role in renal fibrosis and inflammation in CKD. Recently, Smad3 is shown to be a key mediator of downstream TGF- $\beta /$ Smad signaling and mediates renal inflammation and fibrosis via several miRNAs ${ }^{13,15,16}$; however, the involvement of TGF- $\beta / \mathrm{Smad} 3$ on lncRNA expression during renal injury remains unclear. By using the high-throughput RNA sequencing and Smad3 KO mouse models with immune or nonimmune renal diseases, we identified that 151 lncRNAs in UUO and 413 lncRNAs in antiGBM GN were differentially expressed in WT and Smad3 KO kidneys. Among them, 21 novel lncRNAs were coexpressed in both disease models. It is likely that lncRNAs differentially expressed in the UUO kidney may be fibrogenesis related because progressive renal fibrosis in the UUO kidney is Smad3 dependent ${ }^{16,18,30}$; those differentially expressed in anti-GBM GN may be immune and inflammation associated because TGF- $\beta /$ Smad signaling has been shown to play a crucial role in T-cell and macrophage-mediated antiGBM GN. ${ }^{15}$ It is also possible that 21 common lncRNAs that co-expressed in both disease models may be associated with TGF- $\beta /$ Smad3-mediated renal inflammation and/or fibrosis. However, the functional role of these lncRNAs that differentially expressed in anti-GBM GN and UUO nephropathy is largely unknown and requires further studies. 
Increasing evidence shows that there are several relationships between lncRNAs and their associated protein-coding genes. ${ }^{33,34}$ In this study, we showed that the genomic status of 21 common Smad3-dependent IncRNAs included two sense, two intergenic, and 17 intronic lncRNAs (Table 2). Interestingly, some of them were clustered closely within the same chromosome. For example, np_18588 and np_29908 were located in the same intron of inactive phospholipase Clike protein 1, whereas np_18636 and np_5318 were located within the same intron of tyrosine kinase-type cell surface receptor 4 (Erbb4), implying that they may co-express and be regulated by the same transcriptional regulators.

Many ncRNAs, such as miRNAs and small-nucleolar RNAs, exhibit strong conservation across diverse species. Most of the miRNAs have $90 \%$ sequence identity between human and mouse snRNAs conserved at levels between $80 \%$ and $90 \% .{ }^{39}$ However, lncRNAs lack such strong conservation, including Air and Xist. ${ }^{39,40}$ This observation suggests that, unlike mRNAs and miRNAs, which have conserved nucleotides to ensure codon use or target identity, the lack of conservation of lncRNAs may work differently to exert their functions. ${ }^{39-41}$

Because the RNA-Seq was performed with whole kidney samples, this information does not provide any information about where these lncRNAs are expressed. Further studies need to be performed to clarify the spatial and temporal expression patterns of these potential lncRNAs within both normal and diseased kidneys. Moreover, it seems that there were low expression levels of IncRNAs within the kidney because the Pearson correlation in our studies was moderate (values from 0.4 to 0.7 ). This observation was consistent with other studies in which the Pearson correlations of lncRNAs were $<0.6$. $^{42-44}$

In conclusion, our study identifies the Smad3-dependent lncRNAs related to renal inflammation and fibrosis. Results from this study demonstrate, for the first time to our knowledge, that IncRNAs may be involved in TGF- $\beta$ / Smad3-mediated renal inflammation and fibrosis. Further studies to characterize these Smad3-associated lncRNAs should provide valuable information for a better understanding of the insightful mechanisms of TGF- $\beta$-mediated renal inflammation and fibrosis. Moreover, identification of the functional lncRNAs associated with renal inflammation and/or fibrosis may represent a promising research direction into renal disorder and may lead to the development of new lncRNA therapies for kidney diseases.

\section{Supplemental Data}

Supplemental material for this article can be found at http://dx.doi.org/10.1016/j.ajpath.2013.10.007.

\section{References}

1. Eddy AA: Progression in chronic kidney disease. Adv Chronic Kidney Dis 2005, 12:353-365
2. Roberts AB, Tian F, Byfield SD, Stuelten C, Ooshima A, Saika S, Flanders KC: Smad3 is key to TGF-beta-mediated epithelial-tomesenchymal transition, fibrosis, tumor suppression and metastasis. Cytokine Growth Factor Rev 2006, 17:19-27

3. Lan HY, Chung AC: TGF-beta/Smad signaling in kidney disease Semin Nephrol 2012, 32:236-243

4. Yang F, Huang XR, Chung AC, Hou CC, Lai KN, Lan HY: Essential role for Smad3 in angiotensin II-induced tubular epithelialmesenchymal transition. J Pathol 2010, 221:390-401

5. Chung AC, Zhang H, Kong YZ, Tan JJ, Huang XR, Kopp JB, Lan HY: Advanced glycation end-products induce tubular CTGF via TGF-betaindependent Smad3 signaling. J Am Soc Nephrol 2010, 21:249-260

6. Meng XM, Huang XR, Chung AC, Qin W, Shao X, Igarashi P, Ju W Bottinger EP, Lan HY: Smad2 protects against TGF-beta/Smad3mediated renal fibrosis. J Am Soc Nephrol 2010, 21:1477-1487

7. Lorenzen JM, Haller H, Thum T: MicroRNAs as mediators and therapeutic targets in chronic kidney disease. Nat Rev Nephrol 2011, 7: 286-294

8. Kato M, Natarajan R: MicroRNA circuits in transforming growth factor-beta actions and diabetic nephropathy. Semin Nephrol 2012, 32: $253-260$

9. Wei Q, Mi QS, Dong Z: The regulation and function of microRNAs in kidney diseases. IUBMB Life 2013, 65:602-614

10. Chung AC, Yu X, Lan HY: MicroRNA and nephropathy: emerging concepts. Int J Nephrol Renovasc Dis 2013, 6:169-179

11. Lan HY, Chung AC: Transforming growth factor-beta and Smads. Contrib Nephrol 2011, 170:75-82

12. Meng XM, Chung AC, Lan HY: Role of the TGF-beta/BMP-7/Smad pathways in renal diseases. Clin Sci 2013, 124:243-254

13. Qin W, Chung AC, Huang XR, Meng XM, Hui DS, Yu CM, Sung JJ, Lan HY: TGF-beta/Smad3 signaling promotes renal fibrosis by inhibiting miR-29. J Am Soc Nephrol 2011, 22:1462-1474

14. Zhong X, Chung AC, Chen HY, Dong Y, Meng XM, Li R, Yang W, Hou FF, Lan HY: miR-21 is a key therapeutic target for renal injury in a mouse model of type 2 diabetes. Diabetologia 2013, 56:663-674

15. Zhong X, Chung ACK, Chen H-Y, Meng X-M, Lan HY: Smad3mediated upregulation of miR-21 promotes renal fibrosis. J Am Soc Nephrol 2011, 22:1668-1681

16. Chung ACK, Huang XR, Meng X, Lan HY: miR-192 mediates TGFB/Smad3-driven renal fibrosis. J Am Soc Nephrol 2010, 21 1317-1325

17. Brown SD: XIST and the mapping of the X chromosome inactivation centre. Bioessays 1991, 13:607-612

18. Guttman M, Rinn JL: Modular regulatory principles of large noncoding RNAs. Nature 2012, 482:339-346

19. Wang Z, Gerstein M, Snyder M: RNA-Seq: a revolutionary tool for transcriptomics. Nat Rev Genet 2009, 10:57-63

20. Marguerat S, Bahler J: RNA-seq: from technology to biology. Cell Mol Life Sci 2010, 67:569-579

21. Huang XR, Chung AC, Wang XJ, Lai KN, Lan HY: Mice overexpressing latent TGF-betal are protected against renal fibrosis in obstructive kidney disease. Am J Physiol Renal Physiol 2008, 295: F118-F127

22. Huang XR, Chung AC, Zhou L, Wang XJ, Lan HY: Latent TGF-beta1 protects against crescentic glomerulonephritis. J Am Soc Nephrol 2008, 19:233-242

23. Chung AC, Dong Y, Yang W, Zhong X, Li R, Lan HY: Smad7 suppresses renal fibrosis via altering expression of TGF-beta/Smad3regulated microRNAs. Mol Ther 2013, 21:388-398

24. Li R, Rosendahl A, Brodin G, Cheng AM, Ahgren A, Sundquist C, Kulkarni S, Pawson T, Heldin CH, Heuchel RL: Deletion of exon I of SMAD7 in mice results in altered B cell responses. J Immunol 2006, 176:6777-6784

25. Chung AC, Huang XR, Zhou L, Heuchel R, Lai KN, Lan HY: Disruption of the Smad7 gene promotes renal fibrosis and inflammation in unilateral ureteral obstruction (UUO) in mice. Nephrol Dial Transplant 2009, 24:1443-1454 
26. Kong L, Zhang Y, Ye ZQ, Liu XQ, Zhao SQ, Wei L, Gao G: CPC: assess the protein-coding potential of transcripts using sequence features and support vector machine. Nucleic Acids Res 2007, 35: W345-W349

27. Burge SW, Daub J, Eberhardt R, Tate J, Barquist L, Nawrocki EP, Eddy SR, Gardner PP, Bateman A: Rfam 11.0: 10 years of RNA families. Nucleic Acids Res 2013, 41:D226-D232

28. Ovcharenko I, Nobrega MA, Loots GG, Stubbs L: ECR Browser: a tool for visualizing and accessing data from comparisons of multiple vertebrate genomes. Nucleic Acids Res 2004, 32:W280-W286

29. Li R, Chung AC, Dong Y, Yang W, Zhong X, Lan HY: The microRNA miR-433 promotes renal fibrosis by amplifying the TGFB/Smad3-Azin1 pathway. Kidney Int 2013, 84:1129-1144

30. Trapnell C, Pachter L, Salzberg SL: TopHat: discovering splice junctions with RNA-Seq. Bioinformatics 2009, 25:1105-1111

31. Trapnell C, Williams BA, Pertea G, Mortazavi A, Kwan G, van Baren MJ, Salzberg SL, Wold BJ, Pachter L: Transcript assembly and quantification by RNA-Seq reveals unannotated transcripts and isoform switching during cell differentiation. Nat Biotechnol 2010, 28: $511-515$

32. Mortazavi A, Williams BA, McCue K, Schaeffer L, Wold B: Mapping and quantifying mammalian transcriptomes by RNA-Seq. Nat Methods 2008, 5:621-628

33. Zhang Y, Liu XS, Liu QR, Wei L: Genome-wide in silico identification and analysis of cis natural antisense transcripts (cis-NATs) in ten species. Nucleic Acids Res 2006, 34:3465-3475

34. Rinn JL, Chang HY: Genome regulation by long noncoding RNAs. Annu Rev Biochem 2012, 81:145-166

35. Khalil AM, Guttman M, Huarte M, Garber M, Raj A, Morales DR, Thomas K, Presser A, Bernstein BE, van Oudenaarden A, Regev A, Lander ES, Rinn JL: Many human large intergenic noncoding RNAs associate with chromatin-modifying complexes and affect gene expression. Proc Natl Acad Sci U S A 2009, 106:11667-11672
36. Orom UA, Derrien T, Beringer M, Gumireddy $\mathrm{K}$, Gardini A, Bussotti G, Lai F, Zytnicki M, Notredame C, Huang QH, Guigo R, Shiekhattar R: Long noncoding RNAs with enhancer-like function in human cells. Cell 2010, 143:46-58

37. Cabili MN, Trapnell C, Goff L, Koziol M, Tazon-Vega B, Regev A, Rinn JL: Integrative annotation of human large intergenic noncoding RNAs reveals global properties and specific subclasses. Gene Dev 2011, 25:1915-1927

38. Chung AC, Huang XR, Zhou L, Heuchel R, Lai KN, Lan HY: Disruption of the Smad7 gene promotes renal fibrosis and inflammation in unilateral ureteral obstruction (UUO) in mice. Nephrol Dial Transplant 2009, 24:1443-1454

39. Pang KC, Frith MC, Mattick JS: Rapid evolution of noncoding RNAs: lack of conservation does not mean lack of function. Trends Genet 2006, 22:1-5

40. Nesterova TB, Slobodyanyuk SY, Elisaphenko EA, Shevchenko AI, Johnston C, Pavlova ME, Rogozin IB, Kolesnikov NN, Brockdorff N, Zakian SM: Characterization of the genomic Xist locus in rodents reveals conservation of overall gene structure and tandem repeats but rapid evolution of unique sequence. Genome Res 2001, 11:833-849

41. Ponting CP, Oliver PL, Reik W: Evolution and functions of long noncoding RNAs. Cell 2009, 136:629-641

42. Gellert P, Ponomareva Y, Braun T, Uchida S: Noncoder: a web interface for exon array-based detection of long non-coding RNAs. Nucleic Acids Res 2013, 41:e20

43. Petazzi P, Sandoval J, Szczesna K, Jorge OC, Roa L, Sayols S, Gomez A, Huertas D, Esteller M: Dysregulation of the long noncoding RNA transcriptome in a Rett syndrome mouse model. RNA Biol 2013, 10:1197-1203

44. Khaitan D, Dinger ME, Mazar J, Crawford J, Smith MA, Mattick JS Perera RJ: The melanoma-upregulated long noncoding RNA SPRY4-IT1 modulates apoptosis and invasion. Cancer Res 2011, 71: 3852-3862 